\title{
Evaluation of the best solution for the functionalization of photocatalytic, superhydrophobic, and self-cleaning properties on asphalt mixtures
}

\author{
Iran Rocha Segundo ${ }^{1,7^{*}}$, Salmon Landi Jr. ${ }^{2}$, Elisabete Freitas ${ }^{3}$, Cátia Afonso ${ }^{4}$, Manuel F. M. Costa ${ }^{5}$, Behzad Zahabizadeh ${ }^{3}$, \\ Vitor M.C.F. Cunha ${ }^{3}$, Graça Soares ${ }^{6}$, Jorge Santos $^{6}$, Vasco Teixeira ${ }^{7}$, and Joaquim Carneiro ${ }^{7}$ \\ ${ }^{1}$ Civil Engineering Department, University of Minho, Azurém, Guimarães, Portugal \\ ${ }^{2}$ Federal Institute Goiano, Rio Verde - GO, Brazil \\ ${ }^{3}$ University of Minho, ISISE, Department of Civil Engineering, Guimarães, Portugal \\ ${ }^{4}$ IPC - Institute for Polymers and Composites, University of Minho, Guimarães, Portugal \\ ${ }^{5}$ Centre of Physics, University of Minho, Gualtar Campus, Braga, Portugal \\ ${ }^{6}$ Textile Engineering Department, University of Minho, Azurém Campus, Guimarães, Portugal \\ ${ }^{7}$ Centre of Physics, University of Minho, Azurém Campus, Guimarães, Portugal
}

\begin{abstract}
This research aims to develop asphalt mixtures with new capabilities, namely photocatalytic, superhydrophobic, and self-cleaning capabilities. Different solutions were prepared combining nano- $\mathrm{TiO}_{2}$ and micro-PTFE on water, ethyl alcohol, and dimethyl ketone with different concentrations. The solutions were sprayed over the asphalt mixtures, and the photocatalytic and superhydrophobic capabilities were evaluated via decolourization of Rhodamine B dye aqueous solution under simulated solar irradiation and Water Contact Angle (WCA) measurements, respectively. Among the combinations analysed, the best solution was $\mathrm{TiO}_{2}-\mathrm{PTFE}$ with ethyl alcohol $(8 \mathrm{~g} / \mathrm{L})$, which provided a decolourization of about $60 \%$ after having spent $8 \mathrm{~h}$ in light exposure conditions and a superhydrophobic surface with WCA equal to $153^{\circ}$. Thus, this research work aims to contribute to advances in the field of clean technologies, assisting in the transition to the "Green Recovery", which is environmentally friendly, inclusive, and sustainable.
\end{abstract}

\section{Introduction}

Currently, several research works have contributed to advances in clean technologies, assisting in the transition to an innovative socio-economic model identified as "Green Recovery", which is environmentally friendly, inclusive, and sustainable. In this sense, the coatings composed of nano/microparticles applied over asphalt mixtures have been provided new capabilities, such as: photocatalytic, to photodegrade pollutants for air cleaning; superhydrophobic, to improve road safety; and self-cleaning, to avoid slipping problems [1,2]. In general, the photocatalytic capability is developed on asphalt mixtures by the application of semiconductor materials, mainly titanium dioxide $\left(\mathrm{TiO}_{2}\right)$, by using four significant methods: spraying coating, bitumen modification, volume incorporation, and spreading [3]. In addition to being actual recipients of substantial quantities of nanomaterials, these functionalized asphalt mixtures also act as essential tools for leveraging the emerging industrial segment dedicated to producing materials in the form of nanoparticles.

Road pavements are ideal infrastructures to reduce air pollution levels due to their enormous surface area and their proximity to the pollutant gases (mostly from vehicles), especially in urban areas [4]. When functionalized with semiconductor materials, the photocatalytic process on roads is initiated by the electron's excitation from the valence band to the conduction band via photons with proper energy. Then, charge carriers are formed (electrons $\mathrm{e}^{-}$and holes $\mathrm{h}^{+}$). They can react with oxygen and humidity, generating free radicals which can degrade, for example, $\mathrm{NO}_{\mathrm{x}}$ and $\mathrm{SO}_{\mathrm{x}}$.

The functionalization process also endows the material self-cleaning properties, which are related to the degradation of organic compounds, such as oils and greases. This effect can mitigate the decrease of friction caused by these pollutants' contamination over the road surface [5].

On the other hand, the benefits of providing the superhydrophobic capability to asphalt mixtures are mainly to increase water repellence and prevent ice formation. Therefore, the decrease of friction caused by water/ice over the road surface can be reduced. In this specific case, the self-cleaning effect is based on water droplets' ability to carry dirt particles. For this purpose, the literature refers to the application of nano/micromaterials (with a grain size between $6 \mathrm{~nm}$ and $45 \mathrm{~m}$ ) over asphalt mixtures, namely: $\mathrm{TiO}_{2}, \mathrm{ZnO}$ [6], modified- $\mathrm{SiO}_{2}$ [7], modified fluorine-containing polymer

\footnotetext{
*Corresponding author: iran_gomes@hotmail.com
} 
with nano- $\mathrm{CaO}[8]$, Magnesium-Aluminum layered double hydroxides [9], and PTFE [10]. Usually, for this specific functionalization process, solutions (or dispersions) with these particles are prepared and sprayed over the asphalt mixture surface layer $[6,10,11]$.

Thus, the main objective of this research work is to develop asphalt mixtures with photocatalytic, superhydrophobic, and self-cleaning capabilities. To achieve it, different types of solutions were prepared and sprayed over the asphalt mixture AC 10, namely with $\mathrm{TiO}_{2}$ and Polytetrafluoroethylene (PTFE) using water, ethyl alcohol, and dimethyl ketone.

\section{Materials and Methods}

PTFE microparticles with the size of $1 \mu \mathrm{m}$ were purchased from Merck (Algés, Portugal) while $\mathrm{TiO}_{2}$ nanoparticles $(80 \%$ anatase and $20 \%$ rutile, particle size is of about 23 to $28 \mathrm{~nm}$ ) were acquired from Quimidroga (Barcelona, Spain).

First, different types of solutions were prepared with nano- $\mathrm{TiO}_{2}$ and micro-PTFE with water, ethyl alcohol, and dimethyl ketone using two concentrations (4 and $8 \mathrm{~g} / \mathrm{L}$ ). It was not possible to prepare aqueous solutions with PTFE since the particles cannot be dispersed into this medium.

The method of application of the particles was by spraying the solutions over the surface of a conventional asphalt mixture already compacted. The solutions were sprayed over the asphalt mixture samples $\left(8 \mathrm{~mL} / \mathrm{cm}^{2}\right.$ at room temperature) with the dimensions of $25 \times 25 \times 15 \mathrm{~mm}^{3}$ in order to choose the best solution. The asphalt mixture samples were named by an alphanumeric string, which starts by $\mathrm{AC} 10$ to indicate the asphalt concrete, the particle used $\left(\mathrm{TiO}_{2}\right.$ and/or PTFE) and then the solvent used (W - water, ETH - ethyl alcohol, and CET dimethyl ketone), respectively. If both particles, nano$\mathrm{TiO}_{2}$ and micro-PTFE, were combined, at the end of the string, the concentration of the solution was inserted, for example, AC 10 TiO2PTFE-ETH-8g/L. In this case, $4 \mathrm{~g} / \mathrm{L}$ and $8 \mathrm{~g} / \mathrm{L}$ represent $2 \mathrm{~g} / \mathrm{L}$ and $4 \mathrm{~g} / \mathrm{L}$ of each solute, respectively. In case of the solutions with just one type of particles, the concentration was $4 \mathrm{~g} / \mathrm{L}$.

The photocatalytic efficiency was evaluated via Rhodamine B (RhB) degradation following [12,13]. The asphalt mixture samples were immersed into $30 \mathrm{~mL}$ of $\mathrm{RhB}$ aqueous solution with a concentration of $5 \mathrm{ppm}$. The systems were placed inside a box containing a sunlight simulation lamp, located $25 \mathrm{~cm}$ above the samples, resulting in a power intensity of $11 \mathrm{~W} / \mathrm{m}^{2}$. First, the samples were kept in dark condition for $3 \mathrm{~h}$ and then they were irradiated during $8 \mathrm{~h}$. In this way the adsorption and photocatalysis phenomena could be split and the photocatalytic efficiency could be better analysed. To avoid variations in concentration due to the evaporation of $\mathrm{RhB}$ solution, the systems were closed with a transparent plastic film with at least $90 \%$ of transmittance (between 292 and $900 \mathrm{~nm}$ ). The maximum absorption $\left(554 \mathrm{~nm}^{-1}\right)$ of the dye was evaluated as function of the time (using a Shimadzu 3101 PC). The photocatalytic efficiency $(\Phi)$ was calculated with the Equation 1, according to [5].

$$
\Phi(\%)=\left(\frac{A_{o}-A}{A_{o}}\right) \times 100
$$

Where $\mathrm{A}$ and $\mathrm{A}_{0}$ represent the maximum absorbance of RhB solution for time " $\mathrm{t}$ " and 0 hour, respectively.

The Water Contact Angle (WCA) test was carried out to evaluate the wettability of the functionalized asphalt mixtures. Using the equipment OCA 15 plus Dataphysics, 3 readings of $5 \mathrm{~L}$ water drops were carried out at 2 samples for 2 minutes, at room temperature and relative humidity, and the arithmetic mean was calculated. Surfaces with WCA higher than $150^{\circ}$ present superhydrophobic capability $[6,10,11,14]$.

\section{Results and Discussions}

Figure 1 shows the results of the photocatalytic essay. A global examination of these results reveals that the samples only with PTFE (AC 10 PTFE-ETH and AC 10 PTFE-CET) presented a photocatalytic efficiency $21 \%$ higher than AC 10. In addition, the samples with $\mathrm{TiO}_{2}$ showed a photocatalytic efficiency at least $38 \%$ higher than the AC 10 at the end of the test, with the worst performing results for the sample AC 10 TiO2PTFECET $-4 \mathrm{~g} / \mathrm{L}$, which increased the efficiency from $34 \%$ to $47 \%$ (38\% increase), when at least $\mathrm{TiO}_{2}$ is used.

When ketone was used, the highest efficiencies were achieved with only $\mathrm{TiO}_{2}$. Besides that, when the ethyl alcohol was used, the best treatment was AC 10 TiO2PTFE-ETH-8g/L, then AC 10 TiO2-ETH and AC 10 TiO2PTFE-ETH-4g/L. The effect of the solvent on the efficiency of samples with $\mathrm{TiO}_{2}$ in decreasing order of performance was ketone followed by ethyl alcohol and water, with almost the same results.

For the combination of the particles, the increase of the photocatalytic efficiency was $5 \%$ for the ethyl alcohol and $15 \%$ for the ketone, when the concentration increases from $4 \mathrm{~g} / \mathrm{L}$ and $8 \mathrm{~g} / \mathrm{L}$. It can be seen that the best solutions achieved were TiO2-CET and TiO2-PTFE under an ethyl alcohol medium with a concentration of $8 \mathrm{~g} / \mathrm{L}$ (AC 10 TiO2PTFE-ETH-8g/L).

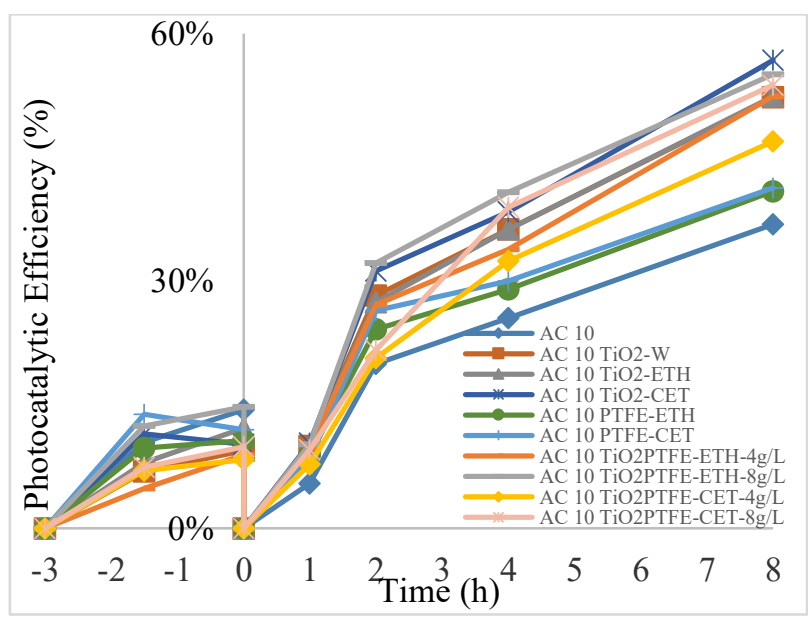

Fig. 1. Photocatalytic efficiency results. 
Figure 2 shows the WCA results. All the treated samples increased WCA when compared to AC 10. The increase was at least $11 \%$ concerning the AC 10 TiO2CET sample. All functionalized asphalt mixtures presented a superhydrophobic capability $\left(\mathrm{WCA}>150^{\circ}\right)$, except $\mathrm{TiO} 2-\mathrm{W}\left(145^{\circ}\right)$ and $\mathrm{TiO} 2-\mathrm{CET}\left(130^{\circ}\right)$, which are overhydrophobic surfaces $\left(120^{\circ}<\right.$ WCA $<$ $\left.150^{\circ}\right)[6,10,11,14]$. Thus, the treatments TiO2-ETH, PTFE-ETH, PTFE-CET, TiO2PTFE-ETH, and TiO2PTFE-CET provided superhydrophobic capability.

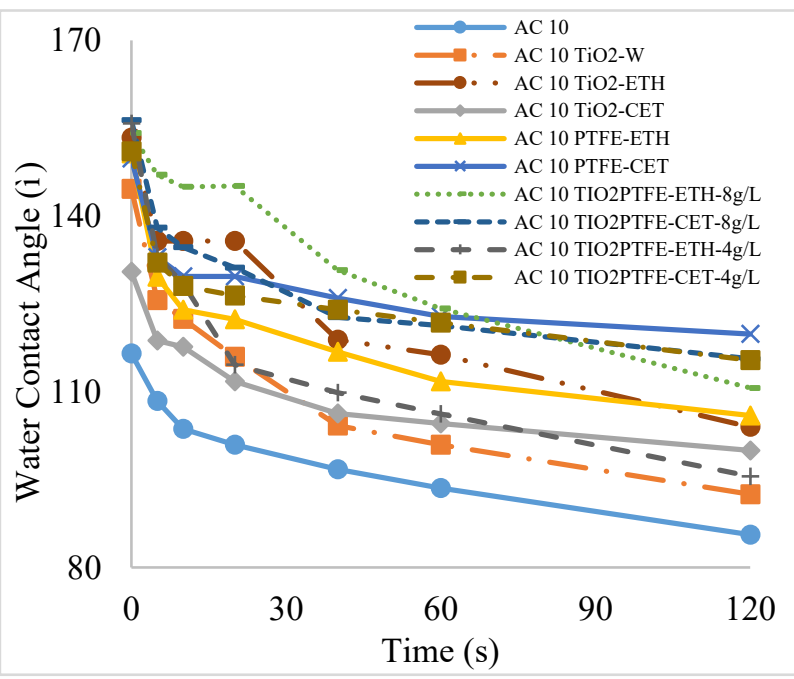

Fig. 2. Water Contact Angle results.

Regarding the effect of the solvents with $\mathrm{TiO}_{2}$, ethyl alcohol led to the best WCA $\left(153^{\circ}\right)$, followed by water $\left(145^{\circ}\right)$. The worst of them was with ketone $\left(130^{\circ}\right)$, as already mentioned before. For the PTFE, both ketone and ethyl alcohol treatments presented similar initial results, but with time, the best of them was with ketone.

The increase in the concentration of TiO2-PTFE was more effective on the ethyl alcohol. For the whole testing, doubling the concentration leads to an increase of the WCA of $13 \%$ and $2 \%$ for ETH and CET, respectively.

When the particles were combined, the initial WCA was similar $\left(155^{\circ}\right)$, except for AC 10 TIO2-PTFE-CET$4 \mathrm{~g} / \mathrm{L}$, that was slightly less $\left(151^{\circ}\right)$. Nevertheless, considering the whole testing duration, it is clear that samples with higher WCA were the AC 10 TIO2PTFE$\mathrm{ETH}-8 \mathrm{~g} / \mathrm{L}$. The average of WCA from 0 to $120 \mathrm{~s}$ increase $36 \%$ when compared to the reference sample. This same functionalized asphalt mixture showed a WCA at $20 \mathrm{~s}$, and a better performance than all the other samples, that dropped the WCA to at least $131^{\circ}$, along this testing time. As explained before, the best results are represented by the highest initial WCAs.

For the same solvent, solutions with $\mathrm{TiO}_{2}$ and PTFE and a concentration of $8 \mathrm{~g} / \mathrm{L}$ delivered the highest WCA. While the results for the solutions with ketone were similar except for $\mathrm{TiO}_{2}$-CET (the worst of them), for ethyl alcohol, the results were dispersed over time.

Thus, the treatment TIO2PTFE-ETH-8g/L was the best solution since it was the best considering the WCA and the second one regarding photocatalysis. The best one regarding the photocatalytic efficiency (AC TiO2-CET) presented the worst WCA for the treated samples. While the second best considering the WCA (AC 10 TiO2PTFEETH-4g/L) was the fifth performance concerning the photocatalysis. Thus, considering both tests, the best solution was TiO2PTFE-ETH with a concentration of 8 $\mathrm{g} / \mathrm{L}$.

\section{Conclusions}

This research aimed to identify the best solution to apply over asphalt mixtures to provide them with new capabilities, specifically photocatalytic, superhydrophobic, and self-cleaning. For this, different types of solutions were sprayed over a conventional asphalt mixture. Then, the functionalized asphalt mixture samples were submitted to dye degradation for photocatalytic efficiency analysis and Water Contact Angle (WCA) test.

The main conclusions were:

- The coatings with at least $\mathrm{TiO}_{2}$ presented a photocatalytic efficiency at least $38 \%$ higher than the reference mixture without any treatment;

- The best solutions considering this aspect were TiO2CET and TiO2PTFE-ETH-8 g/L;

- The treatments TiO2-ETH, PTFE-ETH, PTFE-CET, TiO2PTFE-ETH, and TiO2PTFE-CET provided superhydrophobic capability;

- The best treatment for WCA was TiO2PTFE-ETH $8 \mathrm{~g} / \mathrm{L}$;

- The best solution for both capabilities was TiO2PTFEETH $8 \mathrm{~g} / \mathrm{L}$.

It is important to emphasize that these functionalized asphalt mixtures can degrade gases like $\mathrm{SO}_{\mathrm{x}}$ and $\mathrm{NO}_{\mathrm{x}}$, avoid road accidents by cleaning the dirt particles and degrade oils/greases over their surface, presenting benefits to road safety and the environment. In this sense, the present work showed that one of the potential destinations for the large-scale application of nanomaterials is the civil engineering, especially road construction. Furthermore, this destination assists in promoting the dynamism and commercial progress of nanomaterial and nanotechnology industries.

\section{Acknowledgements}

This work was supported by FCT (Fundação para a Ciência e a Tecnologia) under the frameworks of the Strategic Funding UIDB/04029/2020 and UIDB/04650/2020, and the project PTDC/FIS-MAC/6606/2020. The first author would like to acknowledge the FCT for the Ph.D. scholarship (SFRH/BD/137421/2018).

\section{References}

1. A. Nahvi, M. Sadoughi, A. Arabzadeh, and A. Sassani, J. Comput. Des. Eng. (2018)

2. M. Hassan, L. N. Mohammad, S. Asadi, H. Dylla, and S. Cooper, J. Mater. Civ. Eng. 25, (2012)

3. I. Rocha Segundo, E. Freitas, S. Landi Jr, M. F. M. Costa, and J. O. Carneiro. Coatings 9 (2019). 
4. C. Toro, B. T. Jobson, L. Haselbach, S. Shen, and S. H. Chung, Atmos. Environ. 139, 37 (2016)

5. J. O. O. Carneiro, S. Azevedo, V. Teixeira, F. Fernandes, E. Freitas, H. Silva, and J. Oliveira, Constr. Build. Mater. 38, 594 (2013)

6. I. Rocha Segundo, C. Ferreira, E. F. Freitas, J. O. Carneiro, F. Fernandes, S. L. Júnior, M. F. Costa, S. Landi Júnior, and M. F. Costa. Constr. Build. Mater. 166, 36 (2018)

7. Y. Gao, L. Qu, B. He, K. Dai, Z. Fang, and R. Zhu. Constr. Build. Mater. 191, 270 (2018)

8. J. H. O. Nascimento, P. Pereira, E. Freitas, and F. Fernandes. 7th International Conference on Maintenance and Rehabilitation of Pavements and Technological Control (Auckland, 2012)

9. C. Peng, H. Zhang, Z. You, F. Xu, G. Jiang, S. Lv, and R. Zhang. Constr. Build. Mater. 189, 227 (2018)

10. A. Arabzadeh, H. Ceylan, S. Kim, K. Gopalakrishnan, and A. Sassani. Transp. Res. Rec. J. Transp. Res. Board 2551, 10 (2016)

11. I. Rocha Segundo, S. Landi, E. Freitas, V. Castelo Branco, M. F. M. Costa, and J. Carneiro. EPJ Web Conf. 238, 12012 (2020)

12. W. Zhang, X. Xiao, X. Zeng, Y. Li, L. Zheng, and C. Wan, J. Alloys Compd. 685, 774 (2016)

13. B. Madhukar, J. Wiener, J. Militky, S. Rwawiire, R. Mishra, K. I. Jacob, Y. Wang, B. M. Kale, J. Wiener, J. Militky, S. Rwawiire, R. Mishra, K. I. Jacob, and Y. Wang, Carbohydr. Polym. 150, 107 (2016)

14. I. Rocha Segundo, E. Freitas, V. T. F. C. Branco, S. Landi, M. F. Costa, and J. O. Carneiro. Renew. Sustain. Energy Rev. 151, 111552 (2021) 